Pacific Journal of Mathematic 


\section{BANACH SPACES WITH POLYNOMIAL NORMS}

\section{BRUCE REZNICK}

A Banach space $X$ is said to be in the class $\mathscr{P}_{2 n}$ if, for all elements $x$ and $y,\|x+t y\|^{2 n}$ is a polynomial in real $t$. These spaces generalize $L_{2 n}$ and are precisely those Banach spaces in which linear identities can occur. We shall discuss further properties of $\mathscr{P}_{2 n}$ spaces, often in terms of the permissible polynomials $p(t)=\|x+t y\|^{2 n}$. For each $n$, the set of such polynomials forms a cone. All spaces in $\mathscr{P}_{2}$ are Hilbert spaces. If $X$ is a two-dimensional real space in $\mathscr{P}_{4}$, then it is embeddable in $L_{4}$. This is not necessarily true for spaces with more dimensions or for $\mathscr{P}_{2 n}, n \geqq 3$. The question of embeddability is equivalent to the classical moment problem. All spaces in $\mathscr{P}_{2 n}$ are uniformly convex and uniformly smooth and thus reflexive. They obey generally weaker versions of the Hölder and Clarkson inequalities. Krivine's inequalities, shown to determine embeddability into $L_{p}, p \neq 2 n$, fail in the even case.

1. Introduction. Throughout, we shall consider real Banach spaces, and, except where indicated, $L_{2 n}(Y, \mu)$ with real-valued functions and real scalars. The phrase " $X$ is embeddable in $L_{2 n}$ " is an abbreviation for " $X$ is isometrically isomorphic to a subspace of $L_{2 n}(Y, \mu)$ for some $(Y, \mu)$." Although $\mathscr{P}_{2 n}$ was introduced and motivated in [11], that paper and this one are largely independent.

2. Norm functions. Suppose $X=\left\langle x_{1}, \cdots, x_{m}\right\rangle$ is the real vector space spanned by the $x_{i}$ 's and $\phi$ is a real function of $m$ real variables. Under what circumstances does $\left\|\Sigma u_{i} x_{i}\right\|=\phi\left(u_{1}, \cdots, u_{m}\right)$ make $(X,\|\cdot\|)$ a Banach space? For $\boldsymbol{u}=\left(u_{1}, \cdots, u_{m}\right)$, let $\phi(\boldsymbol{u})=\phi\left(u_{1}, \cdots, u_{m}\right)$. From the standard definition of the norm, it is evident that conditions (A), (B) and (C) are necessary and sufficient. (Here, $t$ is an arbitrary real.)

(A) $\phi(\boldsymbol{u}) \geqq 0$ and $\phi(\boldsymbol{v})=0$ implies $\phi(\boldsymbol{u}) \equiv \dot{\phi}(\boldsymbol{u}+t v)$

(B) $\phi(t \boldsymbol{u})=|t| \dot{\phi}(\boldsymbol{u})$

(C) $\phi(\boldsymbol{u})+\phi(\boldsymbol{v}) \geqq \phi(\boldsymbol{u}+\boldsymbol{v})$.

Condition (C) is cumbersome to verify; the following lemma simplifies matters.

LemmA 1. Conditions (A), (B) and (C) are equivalent to (A), (B) and (D).

(D) $\psi(t)=\phi(\boldsymbol{u}+t \boldsymbol{v})$ is a convex function in $t$ for all $\boldsymbol{u}$ and $\boldsymbol{v}$.

Proof. Assume (A), (B) and (C) and fix $\boldsymbol{u}$ and $\boldsymbol{v}$. Then for $0 \leqq$ 
$\lambda \leqq 1, \lambda \psi\left(t_{0}\right)+(1-\lambda) \psi\left(t_{1}\right)=\lambda \dot{\phi}\left(\boldsymbol{u}+t_{0} \boldsymbol{v}\right)+(1-\lambda) \dot{\phi}\left(\boldsymbol{u}+t_{1} \boldsymbol{v}\right)=\dot{\phi}\left(\lambda \boldsymbol{u}+\lambda t_{0} v\right)+$ $\dot{\phi}\left((1-\lambda) \boldsymbol{u}+(1-\lambda) t_{1} \boldsymbol{v}\right) \geqq \phi\left(\boldsymbol{u}+\left(\lambda t_{0}+(1-\lambda) t_{1}\right) \boldsymbol{v}\right)=\psi\left(\lambda t_{0}+(1-\lambda) t_{1}\right)$. Conversely, assume (A), (B) and (D), then $\dot{\phi}(\boldsymbol{u})+\phi(\boldsymbol{v})=\psi(0)+\psi(1) \geqq$ $2 \psi(1 / 2)=\phi(\boldsymbol{u}+\boldsymbol{v})$.

Observe that it is sufficient to check $\dot{\phi}$ on all two-dimensional subspaces of $X$. For a discussion of a different condition on twodimensional subspaces, see Dor [2]. We shall consider spaces $X$ in $\mathscr{P}_{2 n}$ for which $p(t)=\|x+t y\|^{2 n}$ is a polynomial in $t$ of degree $2 n$. When $p$ is given in this way, we shall tacitly assume that $\|s x+t y\|^{2 n}=$ $s^{2 n} p(t / s)$ for $s \neq 0$ and $\|y\|^{2 n}=\lim _{t \rightarrow \infty} t^{-2 n} p(t)$; that is, (B) is implicit.

Theorem 1. Suppose $p$ is a nonnegative polynomial of degree 2n. Let $X=\langle x, y\rangle$ and define $\|\cdot\|$ on $X$ by $p(t)=\|x+t y\|^{2 n}$. Then $(X,\|\cdot\|)$ is a Banach space if and only if $2 n p(t) p^{\prime \prime}(t)-(2 n-1)\left(p^{\prime}(t)\right)^{2} \geqq$ 0 for all $t$.

Proof. With $\|s x+t y\|^{2 n}$ defined as above, we need verify (A) and (D). Suppose $(X,\|\cdot\|)$ is a Banach space, then $\psi(t)=\|x+t y\|=$ $p(t)^{1 / 2 n}=f(t)$ is convex. If $x$ and $y$ are linearly dependent then $\|x+t y\|=|a+b t|$, and for $p(t)=(a+b t)^{2 n}, 2 n p p^{\prime \prime}=(2 n-1)\left(p^{\prime}\right)^{2}$. If $x$ and $y$ are linearly independent, then $f(t)>0$ and $f$ is convex if and only if $f^{\prime \prime}(t)=(2 n)^{-2}(f(t))^{1-4 n}\left(2 n p(t) p^{\prime \prime}(t)-(2 n-1)\left(p^{\prime}(t)\right)^{2}\right) \geqq 0$.

On the other hand, suppose $2 n p(t) p^{\prime \prime}(t)-(2 n-1)\left(p^{\prime}(t)\right)^{2} \geqq 0$ and $\|\cdot\|$ is defined as above. If $\|s x+t y\|=0$ for $(s, t) \neq(0,0)$ then either $p\left(t_{0}\right)=0$ or $\lim t^{-2 n} p(t)=0$. As the hypothesized condition is translation-invariant, assume $t_{0}=0$ in the first case. Since $p(t) \geqq 0$ we have $p^{\prime}(0)=0$; let $p(t)=a_{k} t^{k}+o\left(t^{k}\right), a_{k} \neq 0, k \geqq 2$, for small $t$. Then $2 n p(t) p^{\prime \prime}(t)-(2 n-1)\left(p^{\prime}(t)\right)^{2}=-a_{k}^{2} k(2 n-k) t^{2 k-2}+o\left(t^{2 k-2}\right)$ hence $k=2 n, p(t)=a_{2 n} t^{2 n}$ and $(X,\|\cdot\|)$ is a valid one-dimensional space. In the second case, let $p(t)=a_{k} t^{k}+o\left(t^{k}\right)$ for $k<2 n, a_{k} \neq 0$ and $t$ large. Then $k=0$ and $(X,\|\cdot\|)$ is again one-dimensional.

Now suppose $p(t)>0$. Let $\boldsymbol{u}=d x+b y, \boldsymbol{v}=c x+a y$ be given; (D) will be satisfied provided $\psi(t)$ is convex, where

$$
\psi^{2 n}(t)=\|d x+b y+t(c x+a y)\|^{2 n}=|c t+d|^{2 n} p((a t+b) /(c t+d)) .
$$

(If $c=d=0$, then $i$ is a constant and so convex). Note that $\psi^{2 n}$ is again a positive polynomial of degree $2 n$ so that $\psi^{\prime \prime}$ is continuous. It suffices, therefore, to check that $\psi^{\prime \prime}(t) \geqq 0$ for $t \neq-d / c$. As above, $\psi^{\prime \prime}(t) \geqq 0$ provided $2 n \psi(t) \psi^{\prime \prime}(t)-(2 n-1)\left(\psi^{\prime}(t)\right)^{2} \geqq 0$. A computation shows that this expression equals $(a d-b c)^{2}(c t+d)^{4 n-4}$ $\left(2 n p(u) p^{\prime \prime}(u)-(2 n-1)\left(p^{\prime}(u)\right)^{2}\right)$, where $u=(a t+b) /(c t+d)$. Thus, if $2 n p p^{\prime \prime}-(2 n-1)\left(p^{\prime}\right)^{2} \geqq 0$ then every is is convex and $(X,\|\cdot\|)$ is a Banach space. 
It follows from Theorem 1 that the two-dimensional spaces in $\mathscr{P}_{2 n}$ are characterized by $p(t)=\|x+t y\|^{2 n}$, and that a study of such polynomials is appropriate. Note also that generators may be chosen to make any computations easier; in general, (D) must be separately verified for each two-dimensional subspace.

3. The cone $P_{2 n}$. Let $P_{2 n}$ consist of all polynomials $p$ of degree $2 n$ for which $p(t) \geqq 0$ and $C_{2 n}(p(t))=2 n p(t) p^{\prime \prime}(t)-(2 n-1)\left(p^{\prime}(t)\right)^{2} \geqq 0$. If $p(t)=\Sigma\left(\begin{array}{c}2 n \\ k\end{array}\right) a_{k} t^{k}$, then

$$
\begin{aligned}
C_{2 n}(p(t))= & 4 n^{2}(2 n-1)\left(\left(\Sigma\left(\begin{array}{c}
2 n \\
k
\end{array}\right) a_{k} t^{k}\right)\left(\Sigma\left(\begin{array}{c}
2 n-2 \\
k
\end{array}\right) a_{k+2} t^{k}\right)\right. \\
& \left.-\left(\Sigma\left(\begin{array}{c}
2 n-1 \\
k
\end{array}\right) a_{k+1} t^{k}\right)^{2}\right)
\end{aligned}
$$

We shall omit the subscript $2 n$ when it is superfluous. As defined, $C_{2 n}(p)$ is a polynomial with nominal degree $4 n-2$; the coefficients for $t^{4 n-2}$ and $t^{4 n-3}$ actually vanish identically.

TheOREM 2. The set $P_{2 n}$ is a closed cone.

Proof. Suppose $p$ is in $P_{2 n}$. Then $C(p) \geqq 0$ and for $\lambda \geqq 0, \lambda p \geqq 0$ and $C(\lambda p)=\lambda^{2} C(p)$ so $\lambda p$ is in $P_{2 n}$. If $p_{i}$ is in $P_{2 n}$, then $p_{1}+p_{2} \geqq 0$ and $C\left(p_{1}+p_{2}\right)=C\left(p_{1}\right)+C\left(p_{2}\right)+2 n p_{1}^{\prime \prime} p_{2}+2 n p_{1} p_{2}^{\prime}-(4 n-2) p_{1}^{\prime} p_{2}^{\prime}$. Since $p_{i} p_{i}^{\prime \prime} \geqq 0$ we have $\left(2 n p_{i} p_{i}^{\prime \prime}\right)^{1 / 2} \geqq(2 n-1)^{1 / 2}\left|p_{i}\right|$ so that $2 n p_{1}^{\prime \prime} p_{2}+2 n p_{1} p_{2}^{\prime \prime}-$ $(4 n-2) p_{1}^{\prime} p_{2}^{\prime}=2 n\left(\left(p_{1}^{\prime \prime} p_{2}\right)^{1 / 2}-\left(p_{1} p_{2}^{\prime \prime}\right)^{1 / 2}\right)^{2}+4 n\left(p_{1} p_{1}^{\prime \prime}\right)^{1 / 2}\left(p_{2} p_{2}^{\prime \prime}\right)^{1 / 2}-(4 n-2)\left|p_{1} p_{2}\right|+$ $(4 n-2)\left(\left|p_{1} p_{2}\right|-p_{1} p_{2}\right) \geqq 0$. Thus, $P_{2 n}$ forms a cone.

Associate $p(t)=\Sigma\left(\begin{array}{c}2 n \\ k\end{array}\right) a_{k} t^{k}$ with the element $\left(a_{0}, \cdots, a_{2 n}\right)$ in $\boldsymbol{R}^{2 n+1}$ and pull back the usual topology. Convergence is then either pointwise or coefficientwise. If $\left\{p_{m}\right\}$ is a sequence of polynomials in $P_{2 n}$ and $p_{m} \rightarrow p$ then $C\left(p_{m}(t)\right) \rightarrow C(p(t))$. Hence $P_{2 n}$ is closed.

By the proof of Theorem 1, if $p(t)$ is in $P_{2 n}$ then so is

$$
(c t+d)^{2 n} p((a t+b) /(c t+d)) \text {. }
$$

For future reference, observe that, if $p_{1}$ and $p_{2}$ are in $P_{2 n}$ and $C\left(\left(p_{1}+p_{2}\right)\left(t_{0}\right)\right)=0$ then $C\left(p_{1}\left(t_{0}\right)\right)=C\left(p_{2}\left(t_{0}\right)\right)=0, p_{1}^{\prime \prime}\left(t_{0}\right) p_{2}\left(t_{0}\right)=p_{1}\left(t_{0}\right) p_{2}^{\prime \prime}\left(t_{0}\right)$ and $p_{1}^{\prime}\left(t_{0}\right) p_{2}^{\prime}\left(t_{0}\right) \geqq 0$.

Since $P_{2 n}$ is a cone, it is natural to study its extreme elements. For $q(t)=(b t+c)^{2 n}, C_{2 n}(q) \equiv 0$. Suppose $q=p_{1}+p_{2}$, with $p_{i}$ in $P_{2 n}$. If $b=0$, then $p_{1}$ and $p_{2}$ must both be nonnegative constants. Suppose $b \neq 0$, then we may normalize $b=1$ so $q(t)=(t+c)^{2 n}$, hence $p_{1}(-c)=$ $p_{2}(-c)=0$. As in the proof of Theorem 1, it follows that $p_{i}(t)=$ $r_{i}(t+c)^{2 n}$ so each $p_{i}$ is a multiple of $q$. We have proved that $(b t+c)^{2 n}$ is 
an extreme element in $P_{2 n}$. Since $P_{2 n}$ is a cone, $\Sigma\left(b_{k} t+c_{k}\right)^{2 n}$ is in $P_{2 n}$. This is to be expected in light of Theorem 1 applied to the subspace of $\ell_{2 n}$ generated by $\left(b_{1}, b_{2}, \cdots\right)$ and $\left(c_{1}, c_{2}, \cdots\right)$.

If $2 n=2, C_{2}\left(a_{2} t^{2}+2 a_{1} t+a_{0}\right)=4\left(a_{0} a_{2}-a_{1}^{2}\right)$ so that $p \geqq 0$ implies $C_{2}(p) \geqq 0$. Hence the extreme elements of $P_{2}$ are precisely $(b t+c)^{2}$. Surprisingly enough, the same is true for $2 n=4$.

THEOREM 3. The extreme functions of $P_{4}$ are $(b t+c)^{4}$; indeed, if $p$ is in $P_{4}$ then $p(t)=\left(b_{0} t+c_{0}\right)^{4}+\left(b_{1} t+c_{1}\right)^{4}+c_{2}^{4}$ for some $b_{i}$ and $c_{i}$.

Proof. Write $p(t)=\Sigma\left(\begin{array}{l}4 \\ k\end{array}\right) a_{k} t^{k}$, then $(48)^{-1} C_{4}(p(t))=\left(a_{2} a_{4}-a_{3}^{2}\right) t^{4}+$ $\left(2 a_{1} a_{4}-2 a_{2} a_{3}\right) t^{3}+\left(a_{0} a_{4}+2 a_{1} a_{3}-3 a_{2}^{2}\right) t^{2}+\left(2 a_{0} a_{3}-2 a_{1} a_{2}\right) t+a_{0} a_{2}-a_{1}^{2}$. If $p\left(t_{0}\right)=0$, then, as before, $p(t)=a_{4}\left(t-t_{0}\right)^{4}$. If $C\left(p\left(t_{0}\right)\right)=0$, then with $q(t)=p\left(t-t_{0}\right), C(q(0))=0$. As the conclusion is invariant under translation, assume $t_{0}=0$. In this case, since $C(p) \geqq 0, a_{0} a_{2}=a_{1}^{2}$ and $a_{0} a_{3}=a_{1} a_{2}$. As $a_{0}=p(0) \neq 0$, let $a_{1}=r a_{0}$, then $a_{2}=r^{2} a_{0}$ and $a_{3}=$ $r^{3} a_{0}$. If $a_{4}=r^{4} a_{0}+s$ then $C(p(t))=s a_{0} t^{2}(r t+1)^{2}$, so $s \geqq 0$ and $p(t)=$ $a_{0}(r t+1)^{4}+s t^{4}$. (In general $p(t)=a_{0}\left(r\left(t-t_{0}\right)+1\right)^{4}+s\left(t-t_{0}\right)^{4}$.) If the degree of $C(p(t))$ is less than four, then by a similar argument, $p(t)=a_{4}(t+r)^{4}+s, s \geqq 0$. Finally, suppose that $C(p(t))$ is a positive quartic and let $p_{\lambda}(t)=p(t)-\lambda$, then $C\left(p_{\lambda}(t)\right)=C(p(t))-4 \lambda p^{\prime \prime}(t)$. Since $p^{\prime \prime}$ is quadratic, and $p p^{\prime \prime}>0,\left(4 p^{\prime \prime}(t)\right)^{-1} C(p(t))$ is continuous, goes to infinity quadratically in $t$, and achieves a minimum $\lambda_{0}>0$ at $t=t_{0}$. Thus $p(t)-\lambda_{0}$ is in $P_{4}, C\left(p_{\lambda_{0}}\left(t_{0}\right)\right)=0$; hence $p(t)=\lambda_{0}+a_{0}\left(r\left(t-t_{0}\right)+1\right)^{4}+$ $s\left(t-t_{0}\right)^{4}$, which may be rewritten as in the conclusion.

By considering $(c t+d)^{4} p((a t+b) /(c t+d))$ instead of $p$, we may replace $c_{2}^{4}$ by $s^{4}(c t+d)^{4}$ for any pre-selected $c$ and $d$. It would be nice if this pattern continued for $2 n \geqq 6$; unfortunately, this is not the case.

THEOREM 4. If $n \geqq 3$ then there exists a polynomial $p$ in $P_{2 n}$ which cannot be written $p(t)=\Sigma\left(b_{k} t+c_{k}\right)^{2 n}$.

Proof. Fix $n$ and let $p(t)=t^{2 n}+t^{2}+1$. A computation shows that $C_{2 n}(p(t))=\left(8 n^{3}-20 n^{2}+12 n\right) t^{2 n}+\left(8 n^{3}-4 n^{2}\right) t^{2 n-2}+(4-4 n) t^{2}+4 n$. Since $n \geqq 3$, each term but $(4-4 n) t^{2}$ is positive. For $|t| \leqq 1,(4-4 n) t^{2}+4 n \geqq 0 ;$ for $|t| \geqq 1,\left(8 n^{3}-4 n^{2}\right) t^{2 n-2}+(4-4 n) t^{2}>$ $\left(8 n^{3}-4 n^{2}-4 n\right) t^{2}>0$. Thus $C_{2 n}(p(t)) \geqq 0$ and $p$ is in $P_{2 n}$.

Suppose $t^{2 n}+t^{2}+1=\Sigma\left(b_{k} t+c_{k}\right)^{2 n}$; from the coefficient of $t^{4}$ and $t^{2}, 0=\Sigma b_{k}^{4} c_{k}^{2 n-4}$ and $1=\left(\begin{array}{c}2 n \\ 2\end{array}\right) \Sigma b_{k}^{2} c_{k}^{2 n-2}$. Since $n \geqq 3$, the first implies that $b_{k} c_{k}=0$ for each $k$, and this contradicts the second. 
The coefficient 1 for $t^{2}$ is not the best possible. The following proposition provides a sharp estimate.

Proposition 1. If $t^{2 n}+\alpha t^{2 k}+1$ is in $P_{2 n}$, then

$$
0 \leqq \alpha \leqq 2 n(2 n-1) c(k, n),
$$

where $(c(k, n))^{n}=(2 k)^{-k}(2 n-2 k)^{k-n}(2 k-1)^{n-2 k}(2 n-2 k-1)^{2 k-n}$.

Outline of proof. Suppose $p_{\alpha}(t)=t^{2 n}+\alpha t^{2 k}+1$ has the largest $\alpha$, then $C_{2 n}\left(p_{\alpha}(t)\right) \geqq 0$ and $C_{2 n}\left(p_{\alpha}\left(t_{0}\right)\right)=0$ for some $t_{0}$. Hence the derivative vanishes at $t_{0}$ as well. This gives two quadratic equations in $\alpha$ which may be solved simultaneously. After eliminating an extraneous solution, the bound is derived.

We see then that there are extreme functions in $P_{2 n}, n \geqq 3$, which are not of the form $(b t+c)^{2 n}$.

Proposition 2. The extreme rays of $P_{6}$ are generated by

$$
(c t+d)^{2 n} f_{\lambda}((a t+b) /(c t+d))
$$

where $f_{\lambda}(t)=t^{6}+6 \lambda t^{5}+15 \lambda^{2} t^{4}+20 \lambda^{3} t^{3}+15 \lambda^{2} t^{2}+6 \lambda t+1$, and $|\lambda| \leqq$ $1 / 2$ or $|\lambda|=1$.

Outline of proof. As in Theorem 3, we consider special cases and then subtract various $(c t+d)^{6}$ 's. Then $f_{\lambda}$ are those polynomials for which $C_{6}\left(f_{2}(0)\right)=0$ and $C_{6}(f)$ is at most quartic.

As Proposition 2 is not directly relevant to the rest of this paper and its proof is tedious, we omit the details. The general question of finding the extreme rays of $P_{2 n}$ for $n \geqq 4$ remains open.

Let $Q_{2 n}$ denote the closure of the cone of polynomials of the form $\sum_{j=1}^{R}\left(b_{j} t+c_{j}\right)^{2 n} ; Q_{2 n} \subseteq P_{2 n}$ with equality if and only if $2 n=2$ or 4 . As any $2 n+2$ distinct $2 n$th powers are linearly dependent, we may assume that $R \leqq 2 n+1$. Suppose $q(t)=\Sigma\left(\begin{array}{c}2 n \\ k\end{array}\right) a_{k} t^{k}$ is in $Q_{2 n}$. Then $q=\lim q_{m}$, where $q_{m}(t)=\sum_{j=1}^{2 n+1}\left(b_{j}^{(m)} t+c_{j}^{(m)}\right)^{2 n}$. Since $\Sigma\left(b_{j}^{(m)}\right)^{2 n} \rightarrow$ $a_{2 n}$ and $\Sigma\left(c_{j}^{(m)}\right)^{2 n} \rightarrow a_{0}$, we may take $\left|b_{j}^{(m)}\right|<M,\left|c_{j}^{(m)}\right|<M$. Thus there exists a convergent subsequence with limit $b_{j}$ and $c_{j}$ so that one may write $q(t)=\sum_{j=1}^{2 n+1}\left(b_{j} t+c_{j}\right)^{2 n}$ for all $q$ in $Q_{2 n}$. Similar considerations apply for the generalization of $Q_{2 n}$ to several variables.

4. Subspaces of $L_{2 n}$. In [11] we showed that $L_{2 n}(Y, \mu)$ is in $\mathscr{P}_{2 n}$, that is, $\|f+t g\|^{2 n}=\int|f+t g|^{2 n} d \mu$ is a polynomial in $t$ for all $f$ and $g$. The converse, as we shall see, is false. Suppose that 
$X=\langle x, y\rangle$ is a two-dimensional space in $\mathscr{P}_{2 n}$, then $p(t)=\|x+t y\|^{2 n}$ is in $P_{2 n}$. Suppose that $X$ is embeddable in $L_{2 n}(Y, \mu)$, then $p(t)=$ $\Sigma\left(\begin{array}{c}2 n \\ k\end{array}\right) a_{k} t^{k}=\int(f+t g)^{2 n} d \mu=\|f+t g\|^{2 n}$. By Hölder's inequality, since $\int f^{2 n} d \mu<\infty$ and $\int g^{2 n} d \mu<\infty, \int f^{2 n-k} g^{k} d \mu<\infty$ so that the integral can be broken up and $a_{k}=\int f^{2 n-k} g^{k} d \mu$. Let $Y_{0}=\{s \in Y: f(s)=0\}, Z=$ $Y-Y_{0}$; let $d \nu=f^{2 n} d \mu$ and $h=g f^{-1}$ on $Z$. Then we have $a_{k}=$ $\int h^{k} d \nu, 0 \leqq k \leqq 2 n-1$, and $a_{2 n}=\int_{Z} h^{2 n} d \nu+\int_{Y_{0}} g^{2 n} d \mu$. If $\Phi(r)=$ $\nu\left(h^{-1}\{(-\infty, r]\}\right)$, then $a_{k}=\int_{-\infty}^{\infty} s^{k} d \Phi$ for $0 \leqq k \leqq 2 n-1$ and $a_{2 n} \geqq$ $\int_{-\infty}^{\infty} s^{2 n} d \Phi$.

Conversely, suppose there exists a nonnegative measure $\Phi$ and $a_{k}$ 's so that $a_{k}=\int_{-\infty}^{\infty} t^{k} d \Phi$ and $a_{2 n} \geqq \int_{-\infty}^{\infty} t^{2 n} d \Phi$. Define $(Y, \mu)$ as follows: $Y=\boldsymbol{R} \cup\left\{p_{0}\right\}, \mu=\Phi$ on $\boldsymbol{R}$ and $\mu\left\{p_{0}\right\}=a_{2 n}-\int_{-\infty}^{\infty} s^{2 n} d \Phi$. Let $(f(s), g(s))=$ $(1, s)$ on $R$ and $(0,1)$ on $\left\{p_{0}\right\}$. Then $\|f+t g\|^{2 n}=\int_{-\infty}^{\infty}(1+s t)^{2 n} d \Phi+$ $\left(a_{2 n}-\int_{-\infty}^{\infty} s^{2 n} d \Phi\right) t^{2 n}=\Sigma\left(\begin{array}{c}2 n \\ k\end{array}\right) t^{k} \int_{-\infty}^{\infty} s^{k} d \dot{\phi}+\left(a_{2 n}-\int_{-\infty}^{\infty} s^{2 n} d \phi\right) t^{2 n}=\Sigma\left(\begin{array}{c}2 n \\ k\end{array}\right) a_{k} t^{k}=$ $p(t)$.

Fortunately, this transforms the embedding problem into the classical moment problem, which has been studied extensively. The complete solution is known, see for example Akhiezer [1] p. 71, and we may combine this solution with the previous discussion to obtain the following theorem.

Theorem 5. Let $X$ be a two-dimensional Banach space in $\mathscr{P}_{2 n}$ with generators $x$ and $y$ and let $p(t)=\|x+t y\|^{2 n}=\Sigma\left(\begin{array}{c}2 n \\ k\end{array}\right) a_{k} t^{k}$. Define the $(n+1) \times(n+1)$ matrix $B=\left(b_{i j}\right)$ by $b_{i j}=a_{i+j}$ for $0 \leqq i, j \leqq n$. Then $X$ is embeddable in $L_{2 n}$ if and only if the matrix $B$ is positive semidefinite. Further, $X$ is embeddable in $L_{2 n}$ if and only if $p$ is in $Q_{2 n}$.

Proof. The positive semidefiniteness of $B$ is equivalent to the solution of the described moment problem. If $p$ is in $Q_{2 n}$ then $X$ is embeddable in $\ell_{2 n}^{2 n+1}$ in the obvious fashion. If $X$ is embeddable in $L_{2 n}$, then by approximating $d \Phi$ by a sequence of point masses, we see that $p$ is in $Q_{2 n}$.

Corollary 6. If $X$ is two-dimensional space in $\mathscr{P}_{4}$, then $X$ is embeddable in $L_{4}$. There are two-dimensional spaces in $\mathscr{P}_{2 n}, n \geqq 3$, which are not embeddable in $L_{2 n}$. 


\section{Proof. Combine Theorems 3, 4 and 5.}

The case for higher dimensions is less clearcut. Professor J. H. B. Kemperman [6] has pointed out, using techniques from [4] and [5], that the analogous moment problem in more than one variable has a solution which requires knowledge of all polynomials $f\left(u_{1}, \cdots, u_{p}\right)$ of total degree $2 n$ which are nonnegative for all real $u_{i}$.

Specifically, one transforms the polynomial $p\left(t_{1}, \cdots, t_{p}\right)=$ $\left\|x_{0}+t_{1} x_{1}+\cdots+t_{p} x_{p}\right\|^{2 n}$ for a space $X=\left\langle x_{0}, \cdots, x_{p}\right\rangle$ into a family of equations $a\left(m_{1}, \cdots, m_{p}\right)=\int \cdots \int t_{1}^{m_{1}} \cdots t_{p}^{m_{p}} d \mu ; m_{1}+\cdots+m_{p}<2 n$, with inequality if $\Sigma m_{i}=2 n$. Suppose $f\left(u_{1}, \cdots, u_{p}\right) \geqq 0$ for all real $u_{i}$ and $f\left(u_{1}, \cdots, u_{p}\right)=\Sigma b\left(m_{1}, \cdots, m_{p}\right) u_{1}^{m_{1}} \cdots u_{p}^{m_{p}}$, where the sum is taken over all $m_{i}, \Sigma m_{i} \leqq 2 n$. Then certainly $\int \cdots \int f\left(u_{1}, \cdots, u_{p}\right) d \mu=$ $\Sigma a\left(m_{1}, \cdots, m_{p}\right) b\left(m_{1}, \cdots, m_{p}\right) \geqq 0$. It turns out this condition holding for all such $f$ is sufficient for the existence of a measure with the desired property.

Since $X$ is real, it is unreasonable to embed $X$ in an $L_{2 n}$ space with complex scalars; one might, however, embed $X$ in an $L_{2 n}(Y, \mu)$ space with real scalars but complex-valued functions. This situation is taken care of by the following theorem.

THEOREM 7. There is an isometry from the space of all complexfunctions in $L_{2 n}(Y, \mu)$, taken with real scalars, into real $L_{2 n}(Z, \nu)$, where $(Z, \nu)$ consists of $2 n+1$ copies of $(Y, \mu)$.

Proof. It is well known that $\ell_{2}^{2}$ is embeddable in any infinitedimensional Banach space. Let $x$ and $y$ be orthogonal generators of $\ell_{2}^{2}$ and let $\bar{x}$ and $\bar{y}$ be their isometric images in $\ell_{2 n}$. Then $\left(t^{2}+u^{2}\right)^{n}=$ $\|t x+u y\|^{2 n}=\|t \bar{x}+u \bar{y}\|^{2 n}=\Sigma\left(b_{k} t+c_{k} u\right)^{2 n}$; by the remarks at the end of $\S 3$, we may say that $\left(t^{2}+u^{2}\right)^{n}=\sum_{k=1}^{2 n+1}\left(b_{k} t+c_{k} u\right)^{2 n}$. Define the mapping $\phi$ from $L_{2 n}(Y, \mu)$ with complex-valued functions to $L_{2 n}(Z, \nu)$ as follows: if $f=g+i h$ is the decomposition into real and imaginary parts, then $\phi(f)=b_{k} g+c_{k} h$ on the $k$ th copy of $(Y, \mu)$. For real $\lambda_{i}, \phi\left(\lambda_{1} f_{1}+\lambda_{2} f_{2}\right)=\lambda_{1} \phi\left(f_{1}\right)+\lambda_{2} \phi\left(f_{2}\right) ;\|\phi(f)\|^{2 n}=\sum_{k=1}^{2 n+1} \int_{Y}\left(b_{k} g+c_{k} h\right)^{2 n} d \mu=$ $\int_{Y}\left(g^{2}+h^{2}\right)^{n} d \mu=\int_{Y}|f|^{2 n} d \mu=\|f\|^{2 n}$ so $\phi$ is an isometry.

We may actually choose $b_{k}$ and $c_{k}$ by: $b_{k}+i c_{k}=a(n) \exp \left(2 \pi k i(n+1)^{-1}\right)$, where $a(n)=2\left(\left(\begin{array}{c}2 n \\ n\end{array}\right)(2 n+1)\right)^{-1 / 2 n}$. Hilbert has proved that $b_{k}$ and $c_{k}$ may be chosen to be rational; see Ellison [3] p. 11 for an extended discussion. In any case, it suffices to consider embeddings into real $L_{2 n}$. 
5. A counterexample. The remaining case for embedding is the three-dimensional one for $\mathscr{P}_{4}$. We shall construct a threedimensional space in $\mathscr{P}_{4}$ which is not embeddable in $L_{4}$. Consequently, there are spaces with arbitrarily large dimensions which are not embeddable in $L_{4}$. This example is drastically simplified from the one appearing in the author's thesis.

Suppose $X=\langle x, y, z\rangle$ and a polynomial $p(u, v)$ with total degree 4 is given. Let $\|\cdot\|$ be defined on $X$ by $\|x+u y+v z\|^{4}=p(u, v)$; $\|t x+u y+v z\|^{4}$ for $t \neq 1$ is defined in the usual way. In view of Lemma 1, we need check (A), (B) and (D) on every two-dimensional subspace of $X$. Conditions (A) and (B) will be automatic. A twodimensional subspace of $X$ is either $\langle y, z\rangle$ or $\langle x+a y+c z, b y+d z\rangle$ for some $a, b, c, d$. Thus, for $f(u, v)=(p(u, v))^{1 / 4}$, it suffices to show that $\psi(t)=f(a+b t, c+d t)$ is convex for all $a, b, c, d$. (We consider $\langle y, z\rangle$ separately.) Adopt the usual convention that $f_{1}(u, v)=$ $(\partial / \partial u) f(u, v), f_{22}(u, v)=\left(\partial^{2} / \partial v^{2}\right) f(u, v)$, etc. Then $\psi^{\prime \prime}(t)=\left(b^{2} f_{11}+2 b d f_{12}+\right.$ $\left.d^{2} f_{22}\right)(a+b t, c+d t)$. Hence it suffices to show that $f_{11} \geqq 0, f_{22} \geqq 0$ and $f_{11} f_{22} \geqq f_{12}^{2}$ at all points in the plane. If we can verify this for $f=p^{1 / 4}$ then $(X,\|\cdot\|)$ will be a Banach space.

TheOREM 8. For $X=\langle x, y, z\rangle$, let $\|t x+u y+v z\|^{4}=t^{4}+$ $6 t^{2}\left(u^{2}+v^{2}\right)+\left(u^{2}+v^{2}\right)^{2}$. Then $(X,\|\cdot\|)$ is a Banach space which is not embeddable in $L_{4}$.

Proof. Note that $\|t x+u y+v z\|>0$ unless $t=u=v=0$ so that (A) is satisfied. On $\langle y, z\rangle,\|u y+v z\|=\left(u^{2}+v^{2}\right)^{1 / 2}$ so $\langle y, z\rangle$ is isometric to $\ell_{2}{ }^{2}$ and (D) is satisfied. In general, let $f=p^{1 / 4}$, then $16 f_{11}=p^{-7 / 4}\left(4 p p_{11}-3 p_{1}^{2}\right), \quad 16 f_{22}=p^{-7 / 4}\left(4 p p_{22}-3 p_{2}^{2}\right)$ and $16 f_{12}=$ $p^{-7 / 4}\left(4 p p_{12}-3 p_{1} p_{2}\right)$. We must show that $4 p p_{i i}-3 p_{i}^{2} \geqq 0$ and that

$$
\begin{aligned}
& \left(4 p p_{11}-3 p_{1}^{2}\right)\left(4 p p_{22}-3 p_{2}^{2}\right)-\left(4 p p_{12}-3 p_{1} p_{2}\right)^{2} \\
& \quad=4 p\left(4 p\left(p_{11} p_{22}-p_{12}^{2}\right)-3 p_{1}^{2} p_{22}+6 p_{1} p_{2} p_{12}-3 p_{2}^{2} p_{11}\right) \\
& \quad=4 p D(p) \geqq 0 .
\end{aligned}
$$

For $p(u, v)=\|x+u y+v z\|^{4}=1+6\left(u^{2}+v^{2}\right)+\left(u^{2}+v^{2}\right)^{2}$ let $w=u^{2}+v^{2}$, then $p=1+6 w+w^{2}, p_{1}=4 u(3+w), p_{2}=4 v(3+w), p_{11}=4\left(3+w+2 u^{2}\right)$, $p_{12}=8 u v, p_{22}=4\left(3+w+2 v^{2}\right)$. Hence

$$
4 p p_{11}-3 p_{1}^{2}=16\left(3\left(1-u^{2}\right)^{2}+v^{2}\left(19+12 u^{2}+u^{4}\right)+v^{4}\left(9+2 u^{2}\right)+v^{6}\right) \geqq 0
$$

and similarly $4 p p_{22}-3 p_{2}^{2} \geqq 0$. Further, $p_{11} p_{22}-p_{12}^{2}=48(w+3)(w+1)$ and $p_{1}^{2} p_{22}-2 p_{1} p_{2} p_{12}+p_{2}^{2} p_{11}=64 w(w+3)^{3}$, hence

$$
\begin{aligned}
D(p) & =192(w+3)(w+1)\left(w^{2}+6 w+1\right)-192 w(w+3)^{3} \\
& =192(w+3)(w-1)^{2} \geqq 0 .
\end{aligned}
$$


Thus $(x,\|\cdot\|)$ is a Banach space.

If $X$ were embeddable in $L_{4}$, then for some $f, g$ and $h, t^{4}+$ $6 t^{2}\left(u^{2}+v^{2}\right)+\left(u^{2}+v^{2}\right)^{2}=\int_{Y}(t f+u g+v h)^{4} d \mu, \quad$ so $\int f^{4}=\int g^{4}=\int h^{4}=$ $\int f^{2} g^{2}=\int f^{2} h^{2}=1, \int g^{2} h^{2}=1 / 3$. The first five equations imply that $f^{2}=g^{2}$ and $f^{\prime}=h^{2} \mu$ - a.e.; this is contradicted by the sixth. Alternatively, in the spirit of the moment problem, $0 \leqq \int_{Y}\left(f^{2}-g^{2}-h^{2}\right)^{2} d \mu=$ $-1 / 3$. Either proof shows that $X$ is not embeddable in $L_{4}$.

One can make a lengthy plausibility argument that the set of polynomials $p(t, u, v)=\|t x+u y+v z\|^{4}$ has 15 degrees of freedom for spaces in $\mathscr{P}_{4}$ and 14 for spaces in $L_{4}$. The last degree of freedom manifests itself here as the coefficient of $u^{2} v^{2}$.

6. Other properties of $\mathscr{P}_{2 n}$. Since $Q_{2 n} \subseteq P_{2 n}$, with strict inclusion for $n \geqq 3$, it is not obvious that spaces in $\mathscr{P}_{2 n}$ are necessarily as "nice" as spaces in $L_{2 n}$. For example, $L_{2 n}(Y, \mu)$ is uniformly convex and uniformly smooth (see Lindenstrauss and Tzafriri [10] p. 127 for definition) and hence reflexive. Hölder's inequality says that, if $\int f^{2 n}=\int g^{2 n}=1$, then $\left|\int f^{k} g^{2 n-k}\right| \leqq 1$ for $0 \leqq k \leqq 2 n$. Thus if $q(t)=$ $1+\sum_{k=1}^{2 n-1}\left(\begin{array}{c}2 n \\ k\end{array}\right) a_{k} t^{k}+t^{2 n}$ is in $Q_{2 n}$, then $\left|a_{k}\right| \leqq 1$; indeed, $1 \geqq a_{k} \geqq r(k)$, where $r(2 j)=0, r(2 j+1)=-1$. Clarkson's inequality states that $\|f+g\|^{2 n}+\|f-g\|^{2 n} \geqq 2\left(\|f\|^{2 n}+\|g\|^{2 n}\right)$; if $q(t)=\sum_{k=0}^{2 n}\left(\begin{array}{c}2 n \\ k\end{array}\right) a_{k} t^{k}$ is in $Q_{2 n}$, then $q(1)+q(-1) \geqq 2\left(q(0)+a_{2 n}\right)$. As a whole, these properties extend to $\mathscr{P}_{2 n}$, although numerical constants are generally weaker.

Koehler [7] defined a $G_{2 n}$ space to be a Banach space on which a $2 n$-fold inner product $\left\langle x_{1}, \cdots, x_{2 n}\right\rangle$ is defined, satisfying certain regularity conditions. In [11] it was shown that $G_{2 n}$ spaces and $\mathscr{P}_{2 n}$ spaces coincide. Koehler [8] proved that $G_{2 n}$ spaces are uniformly convex. That is, $\mathscr{P}_{2 n}$ spaces are uniformly convex and thus reflexive. To prove uniform smoothness and the other regularity conditions we need the analogue to Hölder's inequality.

THEOREM 9. If $p(t)=1+\sum_{k=1}^{2 n-1}\left(\begin{array}{c}2 n \\ k\end{array}\right) a_{k} t^{k}+t^{2 n}$ is in $P_{2 n}$, then there are constants so that $m(k, 2 n) \leqq a_{k} \leqq M(k, 2 n)$.

Proof. Since $p^{1 / 2 n}(t)$ is convex, by the triangle inequality on the space induced by $p,(1-|t|)^{2 n} \leqq p(t) \leqq(1+|t|)^{2 n}$, so for $t \geqq 0,(t-1)^{2 n} \leqq$ $p(t) \leqq(t+1)^{2 n}$. The set of $2 n-1$ equations $\sum_{k=1}^{2 n-1}\left(\begin{array}{c}2 n \\ k\end{array}\right) a_{k} j^{k}=p(j)-1-j^{2 n}$, $1 \leqq j \leqq 2 n-1$, has a Vandermonde determinant, hence $\left(\begin{array}{c}2 n \\ k\end{array}\right) a_{k}$ may be expressed in terms of $p(j)-1-j^{2 n}$. Since $p(j)$ is bounded one 
obtains bounds on $a_{k}$ which are, in general, wildly generous.

Alternatively, a sequence of polynomials with unbounded $\mathrm{a}_{k}$ 's has a subsequence from which can be deduced the existence of $\bar{p}$ in $P_{2 n}$, $\bar{p}(t)=\sum_{k=1}^{2 n-1}\left(\begin{array}{c}2 n \\ k\end{array}\right) \bar{a}_{k} t^{k}$, not all $\bar{a}_{k}$ 's equal to zero. This yields a contradiction.

It follows that the set of all points $\left(a_{1}, \cdots, a_{2 n-1}\right), A$, in $R^{2 n-1}$ so that $1+\sum_{k=1}^{2 n-1}\left(\begin{array}{c}2 n \\ k\end{array}\right) a_{k} t^{k}+t^{2 n}$ is in $P_{2 n}$ forms a closed (Theorem 2) and bounded (Theorem 9) set. Thus functionals, such as $p(1)$, achieve maxima and minima on $A$.

The actual values of $m(k, 2 n)$ and $M(k, 2 n)$ can be found in a few instances. Since $p(t)$ in $P_{2 n}$ implies $p(-t)$ and $t^{2 n} p(1 / t)$ are in $P_{2 n}, m(2 j+1,2 n)=-M(2 j+1,2 n), m(2 n-k, 2 n)=m(k, 2 n)$ and $M(2 n-k, 2 n)=M(k, 2 n)$. As $L_{2 n}$ spaces are in $\mathscr{P}_{2 n}, M(k, 2 n) \geqq 1$ and $m(k, 2 n) \leqq r(k)$. These coefficients are a two-dimensional property; consequently $m(k, 2 n)$ and $M(k, 2 n)$ are already determined for $2 n=2$ or 4 .

In any case, $a_{1}=\lim _{t \rightarrow \infty} t^{-1}(\|x+t y\|-\|x\|)$, so $\left|a_{1}\right| \leqq 1$ and $M(1,2 n)=-m(1,2 n)=1$. Further, $C(p(0))=(2 n)^{2}(2 n-1)\left(a_{0} a_{2}-a_{1}^{2}\right)$ so $a_{2} \geqq 0$ and $m(2,2 n)=0$. The condition in Theorem 9 is, for general $p$ in $P_{2 n}, a_{k} \leqq M(k, 2 n) a_{0}^{1-\alpha} a_{2 n}^{\alpha}$, where $\alpha=k / 2 n$. From the convexity of $x^{\alpha}$, extreme values are attained on extreme elements in $P_{2 n}$. In this way, considering Proposition 2, one can show that $M(3,6)=$ $-m(3,6)=1$ and $M(2,6)=5^{-5 / 3}(1565+496 \sqrt{10})^{1 / 3} \cong 1.000905$. The general problem remains open.

THEOREM 10. If $X$ is in $\mathscr{P}_{2 n}$ then $X$ is uniformly convex, uniformly smooth and so is reflexive.

Proof. The uniform convexity follows from Koehler, or by noting that $\|x\|=\|y\|=1,\|x+y\|=2$ implies $\|x+t y\|=1+t$ for $t \geqq 0$ so $p(t)=(1+t)^{2 n}$ and $\|x-y\|=0$. Since the set of coefficients $A_{\varepsilon}$ for which $\|x\|=\|y\|=1,\|x-y\| \geqq \varepsilon$ is compact, $\|x+y\|$ achieves a maximum, which is strictly less than 2.

For uniform smoothness, let $\|x\|=\|y\|=1$. For $t \leqq \tau$, by Taylor's theorem, $\|x+t y\|+\|x-t y\|=2+(2 n-1)\left(a_{2}-a_{1}^{2}\right) t^{2}+o\left(t^{2}\right)$. Thus $1 / 2(\|x+t y\|+\|x-t y\|)-1 \leqq c \tau^{2}+o\left(\tau^{2}\right)$ so $X$ is uniformly smooth.

If $X$ is any Banach space, suppose $t=\|y\| \geqq\|x\|=1$ and $u=$ $\|x+y\| \geqq\|x-y\|=v$. Then $u+v \geqq 2 t$ so $u^{p}+v^{p} \geqq u^{p}+(2 t-u)^{p} \geqq$ $2 t^{p} \geqq t^{p}+1$. That is, $\|x+y\|^{p}+\|x-y\|^{p} \geqq\|x\|^{p}+\|y\|^{p}$ with equality if and only if $\|x\|=\|y\|=\|x+y\|=\|x-y\|=1$. In this case, 
by the triangle inequality, $\|x+r y\| \equiv 1$ for $|r| \leqq 1$ so $X$ cannot be in $\mathscr{F}_{2 n}$. Thus, by the compactness of $A,\|x+y\|^{2 n}+\|x-y\|^{2 n} \geqq$ $c(n)\left(\|x\|^{2 n}+\|y\|^{2 n}\right)$ for $x$ and $y$ in $X$ in $\mathscr{P}_{2 n}$. Taking $x=0, c(n) \leqq 2$.

THEOREM 11. If $X$ is in $\mathscr{P}_{2 n}$ for $n \leqq 3$ then $\|x+y\|^{2 n}+\|x-y\|^{2 n} \geqq$ $2\left(\|x\|^{2 n}+\|y\|^{2 n}\right)$, but this is not necessary true for $n \geqq 4$.

Proof. For $n \leqq 2, X$ is embeddable in $L_{2 n}$. For $n=3$, let $\|x+t y\|^{6}=\sum_{k=0}^{6}\left(\begin{array}{l}6 \\ k\end{array}\right) a_{k} t^{k}$ then $\|x+y\|^{6}+\|x-y\|^{6}-2\|x\|^{6}-2\|y\|^{6}=$ $30\left(a_{2}+a_{4}\right) \geqq 0$ since $m(2,6)=m(4,6)=0$.

Fix $n \geqq 4$ and set $p_{\varepsilon}(t)=1+\varepsilon\left(t^{2}-3 t^{4}+t^{6}\right)+t^{2 n}$ and $\|x+t y\|^{2 n}=$ $p_{\varepsilon}(t)$, then $\|x+y\|^{2 n}+\|x-y\|^{2 n}-2\left(\|x\|^{2 n}+\|y\|^{2 n}\right)=-2 \varepsilon>0$ for $\varepsilon>0$. A computation shows that $C_{2 n}\left(p_{\varepsilon}(t)\right)=4 n^{2}(2 n-1) t^{2 n-2}+$ $\varepsilon(g(t)+\varepsilon h(t)), \quad$ where $\quad g(t)=4 n^{2}(2 n-1) t^{2 n-2}\left(t^{2}-3 t^{4}+t^{6}\right)+$ $2 n\left(1+t^{2 n}\right)\left(2-36 t^{2}+30 t^{4}\right)-4 n(2 n-1) t^{2 n-1}\left(2 t-12 t^{3}+6 t^{5}\right)$ and $h(t)=$ $2 n\left(t^{6}-3 t^{4}+t^{2}\right)\left(30 t^{4}-36 t^{2}+2\right)-(2 n-1)\left(6 t^{5}-12 t^{3}+2 t\right)^{2}$.

As $n \geqq 4$, the highest order term of $g+\varepsilon h$ is

$$
2 n\left(4 n^{2}-26 n+42\right) t^{2 n+4},
$$

there exist $\varepsilon_{0}$ and $R$ so that for $0 \leqq \varepsilon \leqq \varepsilon_{0}$ and $|t|>R,(g+\varepsilon h)(t) \geqq 0$ and thus $C_{2 n}\left(p_{\varepsilon}(t)\right)>0$. As $(g+\varepsilon h)(0)=4 n$, for $0 \leqq \varepsilon \leqq \varepsilon_{0}$ and $|t|<\delta$ or $|t|>R, C_{2 n}\left(p_{\varepsilon}(t)\right)>0$. On the remaining (compact) set, $t^{2 n-2}$ is positive and $|g|+\varepsilon_{0}|h|$ is bounded, so for some further reduced range of $\varepsilon, C_{2 n}\left(p_{\varepsilon}\right)>0$ and $p_{\varepsilon}$ is in $P_{2 n}$.

For $n=4$ take $\varepsilon=.04$, then $p_{\varepsilon}(t)=t^{8}+.04 t^{6}-.12 t^{4}+.04 t^{2}+1$. A direct computation shows that $C_{8}\left(p_{\varepsilon}(t)\right)=64\left(t^{12}+1\right)+11.5392\left(t^{10}+t^{2}\right)+$ $9.68\left(t^{8}+t^{4}\right)+447.9104 t^{6}$. If we factor out $.64 t^{6}$ and let $u=t^{2}+t^{-2}$, then we obtain $u^{3}-18.03 u^{2}+12 u+735.92=q(u)$. (The range for $t^{2}+t^{-2}$ is $u \geqq 2$.) Clearly $q(2)>0$, and $q$ achieves its minimum when $u=u_{0}=6.01+\sqrt{32.1201} \cong 11.67$. Since $q\left(u_{0}\right) \cong 9.79>0, C(4) \leqq$ 1.96. This bound is not sharp. This example also shows that $m(4,8)<0$.

The question of describing spaces dual to spaces in $\mathscr{F}_{2 n}$ also remains open. Indeed it is false, in general, that the dual space to a subspace of $L_{p}(Y, \mu)$ is necessarily embeddable in $L_{q}, p^{-1}+q^{-1}=2$. For example, if $p=2 n /(2 n-1), x=(1,1,0), y=(1,0,1)$ and $X$ is the subspace of $\ell_{p}^{3}$ generated by $x$ and $y$, then $X^{*}$ is not even in $\mathscr{P}_{2 n}$, let alone $L_{2 n}$. We omit the proof.

7. Krivine inequalities. Krivine [9] has described necessary and sufficient conditions for a space to be embeddable in $L_{p}$ provided $p$ is not an even integer. Krivine's proof does not apply when $p=2 n$ because it involves the Taylor series remainder of $\cos x$. Theorem 12 discusses this case and provides an underlying reason for this 
failure when viewed in conjunction with Corollary 6 .

THEOREM (Krivine). If $2 r-2<p<2 r \leqq 4 k$ then a necessary and sufficient condition for $X$ to be embeddable in $L_{p}$ is that (1) holds for all elements $x_{i}$ and all choices of real scalars $r_{i}$ with $\Sigma r_{i}=0$. The sum is taken as the $i_{j}$ 's range independently from 1 to $m$ and as the $\varepsilon_{j}$ 's range over all choices of sign \pm 1 . The sum has $m^{2 k} 2^{2 k-1}$ terms.

$$
(-1)^{r} \sum_{i_{1}=1}^{m} \cdots \sum_{i_{2 k}=1}^{m} r_{i_{1}} \cdots r_{i_{2 k}} \sum_{\varepsilon_{j}}\left\|x_{i_{1}}+\varepsilon_{2} x_{i_{2}}+\cdots+\varepsilon_{2 k} x_{i_{2 k}}\right\|^{p} \geqq 0 \text {. }
$$

THEOREM 12. If $4 k>2 n$ and $X$ is in $\mathscr{P}_{2 n}$, then the sum in (1), taken with $p=2 n$, is identically zero.

Proof. By Theorem 11 in [11], it suffices to verify any linear identity on one space in $\mathscr{P}_{2 n}$, say $C$. Since in (1) all elements are combined with real coefficients, by Theorem 7 , we may embed $C$ isometrically in $\boldsymbol{R}$. It therefore suffices to check that (2) holds in $\boldsymbol{R}$.

$$
\sum_{i_{1}=1}^{m} \cdots \sum_{i_{2 l}=1}^{m} r_{i_{1}} \cdots r_{i_{2 k}} \sum_{ \pm}\left(t_{i_{1}} \pm t_{i_{2}} \pm \cdots \pm t_{i_{2 k}}\right)^{2 n}=0 .
$$

Because of the signs in the inner sum, we may rewrite this in the form $\sum_{j} d_{j} t_{i_{1}}^{\pi j^{(1)}} \cdots t_{i_{2 k}}^{\pi_{2(2 l)}}$, where $j$ indexes all partitions of $2 n$ into $2 k$ even integers and $d_{j}$ is the positive multinomial coefficient. If we now exchange the order of summation, then (2) becomes (3).

$$
\sum_{j} d_{j} \prod_{s=1}^{2 k}\left(\sum_{i_{s}=1}^{m} \boldsymbol{r}_{i_{s}} \boldsymbol{t}_{i_{s}^{j}}^{j^{(s)}}\right)=0
$$

Fix $j$; since $4 k>2 n$, at least one of the $\pi_{j}(s)$ 's is zero. Thus, one term in the product is $\Sigma r_{j}=0$, each term in the sum vanishes and (3) is verified.

For $2 n \geqq 4$, there are spaces in $\mathscr{P}_{2 n}$ which are not embeddable in $L_{4}$, so that Krivine's inequalities do not extend. For $4 k=2 n$ and $X=L_{2 n}(Y, \mu)$, it is not hard to show that the left hand side of (1) becomes $\left(\int \Sigma r_{i} x_{i}^{2} d \mu\right)^{2 k}$ which is nonnegative. If, on the other hand, $X$ is the space in Theorem $8, x_{1}=x, x_{2}=y, x_{3}=z, r_{1}=-2, r_{2}=r_{3}=$ 1 , then $\sum_{1}^{3} \sum_{1}^{3} r_{i} r_{j} \Sigma\left\|x_{i} \pm x_{j}\right\|^{4}=-16$. It is possible that a careful study of Krivine's inequality for such borderline cases could lead to an embedding theorem for $L_{p}, p=2 n$.

ACKNOwledgments. This work grew out of the author's Ph.D. thesis, produced at Stanford University under the direction of Per Enflo. The author expresses his gratitude both to Professor Enflo and to Professor J. H. B. Kemperman, who kindly answered a written 
inquiry in great detail.

\section{REFERENCES}

1. Naum Akhiezer, The Classical Moment Problem, Oliver and Boyd, London, 1965.

2. Leonard Dor, Potentials and isometric embeddings in $L_{1}$, Israel J. Math., 24 (1976), 260-268.

3. W. J. Ellison, Waring's problem, Amer. Math. Monthly, 78 (1971), 10-36.

4. J. H. B. Kemperman, On the sharpness of Tchebycheff type inequalities, Indag. Math., 27 (1965), 554-601.

5. - On a class of moment problems, Proceedings of the Sixth Berkeley Symposium on Mathematical Statistics and Probability, vol. II, pp. 101-126, University of California Press, 1972.

6. - personal correspondence, Sept. 2, 1977.

7. Donald Koehler, $G_{2 n}$ spaces, Trans. Amer. Math. Soc., 150 (1970), 507-518.

8. The analytic properties of $G_{2 n}$ spaces, Proc. Amer. Math. Soc., 35 (1972), 201-206.

9. Jean-Louis Krivine, Plongement des espaces normés dans $L^{p}$, C. R. Acad. Sci. (Paris), 261 (1965), 4307-4310.

10. Joram Lindenstrauss, and Lior Tzafriri, Classical Banach Spaces, Lecture Notes in Mathematics No. 338, Springer-Verlag, New York, 1973.

11. Bruce Reznick, Banach spaces which satisfy linear identities, Pacific J. Math., 74 (1978), 221-233.

Received October 14, 1977.

DUKE UNIVERSITY

DURHAM, NC 27706 



\section{PACIFIC JOURNAL OF MATHEMATICS}

\section{EDITORS}

DoNALD BABBITT (Managing Editor)

University of California

Los Angeles, California 90024

HUGo Rossi

University of Utah

Salt Lake City, UT 84112

C. C. MOORE and ANDREW OGG

University of California

Berkeley, CA 94720
J. DUGUNDJI

Department of Mathematics University of Southern Californı Los Angeles, California 90007

R. Finn and J. Milgram Stanford University Stanford, California 94305

\section{ASSOCIATE EDITORS}

E. F. BECKENBACH

B. H. Neumann
F. WOLF

K. YOSHIDA

\section{SUPPORTING INSTITUTIONS}

UNIVERSITY OF BRITISH COLUMBIA CALIFORNIA INSTITUTE OF TECHNOLOGY UNIVERSITY OF CALIFORNIA MONTANA STATE UNIVERSITY UNIVERSITY OF NEVADA, RENO NEW MEXICO STATE UNIVERSITY OREGON STATE UNIVERSITY UNIVERSITY OF OREGON
UNIVERSITY OF SOUTHERN CALIFORNIA STANFORD UNIVERSITY UNIVERSITY OF HAWAII UNIVERSITY OF TOKYO UNIVERSITY OF UTAH WASHINGTON STATE UNIVERSITY UNIVERSITY OF WASHINGTON 


\section{Pacific Journal of Mathematics}

Vol. 82, No. $1 \quad$ January, 1979

Werner Bäni, Subspaces of positive definite inner product spaces of countable dimension ...................................... 1

Marilyn Breen, The dimension of the kernel of a planar set..............

Kenneth Alfred Byrd, Right self-injective rings whose essential right ideals

are two-sided

Patrick Cousot and Radhia Cousot, Constructive versions of Tarski's fixed

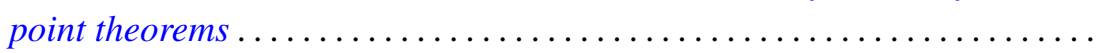

Ralph S. Freese, William A. Lampe and Walter Fuller Taylor, Congruence lattices of algebras of fixed similarity type. $I \ldots \ldots \ldots \ldots \ldots \ldots \ldots$

Cameron Gordon and Richard A. Litherland, On a theorem of Murasugi .....

Mauricio A. Gutiérrez, Concordance and homotopy. I. Fundamental

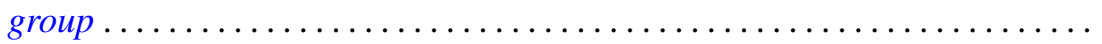

Richard I. Hartley, Metabelian representations of knot groups .............

Ted Hurley, Intersections of terms of polycentral series of free groups and free

Lie algebras ........................................

Roy Andrew Johnson, Some relationships between measures ............ 117

Oldřich Kowalski, On unitary automorphisms of solvable Lie algebras .......

Kee Yuen Lam, $K O$-equivalences and existence of nonsingular bilinear

maps...................................................

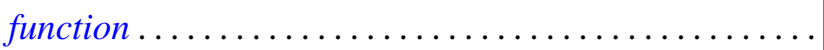

Robert A. Messer and Alden H. Wright, Embedding open 3-manifolds in compact 3-manifolds ............................

Gerald Ira Myerson, A combinatorial problem in finite fields. I . .

James Nelson, Jr. and Mohan S. Putcha, Word equations in a band of paths.

Baburao Govindrao Pachpatte and S. M. Singare, Discrete generalized Gronwall inequalities in three independent variables . .

William Lindall Paschke and Norberto Salinas, $C^{*}$-algebras associated with free products of groups ........................

Bruce Reznick, Banach spaces with polynomial norms ....

David Rusin, What is the probability that two elements of a finite group

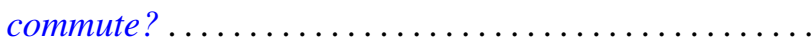

M. Shafii-Mousavi and Zbigniew Zielezny, On hypoelliptic differential operators of constant strength ...

Joseph Gail Stampfli, On selfadjoint derivation ranges .... . . .

Robert Charles Thompson, The case of equality in the matrix-valued triangle

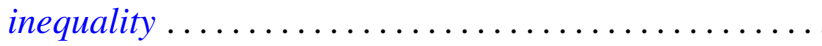

Marie Angela Vitulli, The obstruction of the formal moduli space in the negatively graded case. 